\title{
Thoracentesis outcomes: a 12-year experience
}

\author{
Mark J Ault, ${ }_{1}^{1}$ Bradley T Rosen, ${ }_{1}^{1}$ Jordan Scher, ${ }^{2}$ Joe Feinglass, ${ }^{2}$ Jeffrey H Barsuk ${ }^{2}$
}

- Additional material is published online only. To view please visit the journal online (http://dx.doi.org/10.1136/ thoraxjn-2014-206114)

${ }^{1}$ Division of General Internal Medicine, Cedars-Sinai Medical Center, Los Angeles, California, USA

${ }^{2}$ Department of Medicine, Northwestern University Feinberg School of Medicine, Chicago, Illinois, USA

\section{Correspondence to}

Dr Jeffrey $H$ Barsuk, Division of Hospital Medicine, Department of Medicine, Northwestern University Feinberg School of Medicine, 211 E. Ontario St, Suite 717, Chicago, IL 60611, USA; jbarsuk@nmh.org

Received 31 July 2014 Revised 6 October 2014 Accepted 16 October 2014 Published Online First 5 November 2014

\section{CrossMark}

To cite: Ault MJ, Rosen BT, Scher J, et al. Thorax 2015;70:127-132.

\section{ABSTRACT \\ Background Despite a lack of evidence in the} literature, several assumptions exist about the safety of thoracentesis in clinical guidelines and practice patterns. We aimed to evaluate specific demographic and clinical factors that have been commonly associated with complications such as iatrogenic pneumothorax, reexpansion pulmonary oedema (REPE) and bleeding. Methods We performed a cohort study of inpatients who underwent thoracenteses at Cedars-Sinai Medical Center (CSMC) from August 2001 to October 2013. Data were collected prospectively including information on volume of fluid removed, procedure side, whether the patient was on positive pressure ventilation, number of needle passes and supine positioning. latrogenic pneumothorax, REPE and bleeding were tracked for $24 \mathrm{~h}$ after the procedure or until a clinical question was reconciled. Demographic and clinical characteristics were obtained through query of electronic medical records.

Results CSMC performed 9320 inpatient thoracenteses on 4618 patients during the study period. There were 57 (0.61\%) iatrogenic pneumothoraces, $10(0.01 \%)$ incidents of REPE and $17(0.18 \%)$ bleeding episodes. latrogenic pneumothorax was significantly associated with removal of $>1500 \mathrm{~mL}$ fluid $(p<0.0001)$, unilateral procedures $(p=0.001)$ and more than one needle pass through the skin $(p=0.001)$. For every $1 \mathrm{~mL}$ of fluid removed there was a $0.18 \%$ increased risk of REPE (95\% Cl $0.09 \%$ to $0.26 \%$ ). There were no significant associations between bleeding and demographic or clinical variables including International Normalised Ratio, partial thromboplastin time and platelet counts. Conclusions Our series of thoracenteses had a very low complication rate. Current clinical guidelines and practice patterns may not reflect evidence-based best practices.

Pleural effusions are commonly encountered in hospitalised patients and up to 173000 thoracentesis procedures are performed annually in the USA. ${ }^{1}{ }^{2}$ Complications from thoracentesis include pneumothorax, re-expansion pulmonary oedema (REPE) and bleeding. These complications increase morbidity, prolong length of stay and escalate healthcare costs. ${ }^{3}{ }^{4}$ Despite a lack of supporting evidence in the literature, common assumptions regarding thoracentesis safety guidelines include: (1) avoiding bilateral procedures or procedures on patients receiving positive pressure ventilation due to increased risk of catastrophic complications; ${ }^{15-7}$ (2) limiting fluid removal to $<1500 \mathrm{~mL}$ to avoid REPE; ${ }^{8}$ and (3) not performing procedures on patients with coagulopathy or low platelets without first administering blood products. ${ }^{8} 9$

\section{Key messages}

What is the key question?

- Are common assumptions regarding thoracentesis safety guidelines accurate including:

- Avoiding bilateral procedures or procedures on patients receiving positive pressure ventilation due to increased risk of catastrophic complications;

- Limiting fluid removal to less than $1500 \mathrm{~mL}$ to avoid re-expansion pulmonary oedema; and

- Not performing procedures on patients with coagulopathy or low platelets without first administering blood products?

\section{What is the bottom line?}

- The common assumptions about the safety of thoracentesis procedures are based on weak evidence and may adversely affect patient care.

\section{Why read on?}

- We report a large series of over 9300 thoracentesis procedures and their complications that refute several commonly held assumptions.

Thoracenteses have been a core procedure performed by dedicated proceduralists at Cedars-Sinai Medical Center (CSMC) in Los Angeles for over 12 years. As a standard part of quality assurance, CSMC documents complications from thoracenteses performed in their procedure centre. Northwestern University investigators (JS, JF, JHB) evaluated these data independently to look for associations between procedure complications and patient characteristics. This study reports findings of this collaboration and had three major aims: (1) to evaluate whether specific demographics, clinical factors or procedure techniques were associated with complications including pneumothorax; (2) to evaluate whether REPE was associated with volume of effusion removed; and (3) to evaluate whether coagulopathy or thrombocytopenia increased the risk of thoracentesis-related bleeding.

\section{MATERIALS AND METHODS Design}

We performed a cohort study of inpatients who underwent thoracenteses at CSMC from August 2001 to October 2013. CSMC is a tertiary care academic medical centre with 896 inpatient beds. The procedure service prospectively followed 
patients for complications up to $24 \mathrm{~h}$ after the procedure was completed, and we evaluated these cases for factors associated with these complications. The CSMC and Northwestern University Institutional Review Boards approved this study.

\section{Procedure protocol and registry complication data}

Inpatients at CSMC were referred to the procedure service for thoracenteses by physicians directly involved in each patient's care. The thoracenteses in this study were all performed or supervised by a single clinician/author (MJA) who is the medical director of the procedure service. Procedures were performed in a dedicated suite or at the patients' bedside. Portable ultrasound was routinely used for fluid localisation through site marking (not real-time) and a safety-tipped needle/catheter was used in all cases. Fluid was removed by manual hand pumping. Aspirations were done with a $10 \mathrm{~mL}$ syringe to optimise the ability to detect resistance changes. Ultrasound was used to monitor the progress of fluid removal by intermittently interrogating the pleural space throughout the procedure. Attempts were made in all cases to remove all of the fluid present in the pleural space. However, if resistance to aspiration became notable relative to baseline resistance and/or the patient became symptomatic with chest tightness, cough or pain referred to the upper chest or neck, thoracentesis was terminated. If residual fluid was present in these circumstances, the possibility of trapped lung was noted. Vacuum bottles were used for fluid removal only in rare circumstances. Chest X-rays were only ordered after the procedure if there was a change in patient symptoms or it was clinically necessary to document lung re-expansion. Patients were not treated with blood product administration for coagulopathy or low platelets before the procedure was performed.

Data were collected prospectively by the proceduralist (MJA) at the time of the procedure, including: volume of fluid removed, procedure side, whether the patient was on invasive or non-invasive positive airway pressure ventilation, number of needle passes and supine positioning. Patients were followed for complications up to $24 \mathrm{~h}$ after the procedure or until a clinical question of a complication was reconciled. Recorded complications included pneumothorax, REPE, haemothorax, site bleeding or haematoma, splenic laceration and vasovagal reactions. All data were recorded in a hand-held database (HanDbase, DDH Software).

Patients were considered to have a pneumothorax based on follow-up chest X-ray findings or if the operator began aspirating significant amounts of air with an absence of lung sliding on bedside ultrasound. In cases where it was unclear if the patient had trapped lung or pneumothorax, the diagnosis of pneumothorax was assigned. Resultant air in the pleural space was considered to be a result of trapped lung if radiographically the air identically conformed to the prior fluid shadow, air was noted in a non-gravity-dependent location only, or if there was residual fluid remaining by ultrasound that could not be aspirated due to excessive negative pressure required. Patients were diagnosed with REPE if there was acute decompensation (increased work of breathing or oxygen needs) within the first $24 \mathrm{~h}$ of the procedure and unilateral pulmonary oedema on the chest X-ray or isolated unilateral pulmonary oedema with lack of another more likely diagnosis. Haemothorax was diagnosed if a patient had rapid reaccumulation of pleural fluid with a drop in haemoglobin $(2 \mathrm{~g} / \mathrm{dL})$ or serosanguinous or bloody fluid was discovered on a follow-up thoracentesis. Bleeding complications were combined into one category entitled haemothorax/bleeding/haematoma. Pneumothorax, REPE, haemothorax, site bleeding, haematoma, splenic laceration and vasovagal reactions were combined into an 'any complications' category for further analysis.

We also performed a query of the electronic medical record on patient demographic and clinical information for the admission during which the thoracentesis was performed including: age, sex, body mass index (BMI), International Normalised Ratio (INR), partial thromboplastin time (PTT), platelet counts $\left(10^{3} / \mu \mathrm{L}\right)$ and International Classification of Diseases, 9th Revision (ICD-9) codes. Our query for laboratory data retrieved the closest laboratory entry up to $48 \mathrm{~h}$ before the procedure. If no laboratory data were available before the procedure, the query searched for laboratory data up to $24 \mathrm{~h}$ after the procedure. We used ICD-9 diagnosis codes to calculate patients' Charlson scores (a severity of illness indicator based on 19 chronic disease comorbidities which predicts 1-year mortality for hospitalised medical patients). ${ }^{10} 11$

\section{Statistical analysis}

We used random effects Poisson regression to model the likelihood of either iatrogenic pneumothorax or of any complications. Poisson regression was used because all dependent variables were rare counted events. Random effects Poisson regression adjusts SEs for multiple procedures for individual patients (clustering) and estimates an incidence rate ratio that is closer to the relative risk than the OR when the incidence is low. We first performed a univariate analysis of associations between any complications or pneumothorax and patients' age, sex, BMI, Charlson score, INR, PTT, platelet counts, fluid volume removed, procedure side (bilateral or unilateral), whether the patient was on invasive or non-invasive or mechanical ventilation, number of needle passes and supine positioning. Only univariate associations at the $\mathrm{p} \leq 0.1$ level were included in further multivariate analysis.

Based on univariate findings described below, the model of any complication included BMI, PTT, volume removed, bilateral procedures and number of needle passes, while the iatrogenic pneumothorax model included sex, BMI, PTT, volume removed, bilateral procedures, number of needle passes and supine position. There were too few outcomes to perform separate analyses of REPE and haemothorax/bleeding/haematoma. However, because of assumed clinical risk, we did perform univariate Poisson regression models for the association of REPE with fluid volume removed and the association of patients' INR, PTT and platelet counts with the likelihood of haemothorax/ bleeding/haematoma.

\section{RESULTS}

\section{Incidence of complications and risk factors}

The CSMC procedure service performed 9320 inpatient thoracentesis procedures for 4618 patients during the study period. There were 3796 bilateral procedures performed. Unilateral thoracenteses were performed on 2426 patients, 2192 had two procedures, (coincidentally) 2192 also had three procedures, 916 had four procedures and one patient had 27 procedures. Of the 9320 procedures, $91(0.98 \%)$ had any complication (including 5 patients who experienced 2 complications). There were $57(0.61 \%)$ iatrogenic pneumothoraces, 10 (0.01\%) REPE, $17(0.18 \%)$ bleeding episodes of which $5(0.05 \%)$ were haemothoraces, $1(0.01 \%)$ splenic laceration and $6(0.06 \%)$ vasovagal reactions. Patient clinical and demographic characteristics by procedure and complications are shown in table 1 . Charlson score data were missing in 406/9320 (4.4\%) patients, four of whom had any complication and one had a pneumothorax; INR data were missing in 415/9320 (4.5\%) patients, four of 
Table 1 Patient clinical and demographic characteristics of thoracentesis procedures and outcomes

\begin{tabular}{|c|c|c|c|c|c|}
\hline & $\begin{array}{l}\text { All procedures } \\
(\mathrm{N}=9320)\end{array}$ & $\begin{array}{l}\text { Procedures with } \\
\text { any complication } \\
(\mathrm{N}=91)\end{array}$ & p Value* & $\begin{array}{l}\text { Procedures with } \\
\text { pneumothorax } \\
(\mathrm{N}=57)\end{array}$ & p Value* \\
\hline Age (years), n (\%) & & & 0.46 & & 0.74 \\
\hline$<60$ & $2194(23.77)$ & $26(28.57)$ & & $13(22.81)$ & \\
\hline $60-75$ & 2906 (31.48) & $22(24.18)$ & & $17(29.82)$ & \\
\hline$>75$ & $4220(45.72)$ & $43(47.25)$ & & $27(47.37)$ & \\
\hline Male, n (\%) & $4656(50.44)$ & $52(57.14)$ & 0.19 & $35(61.40)$ & 0.10 \\
\hline BMI category, n (\%) & & & 0.02 & & 0.06 \\
\hline Underweight $<18.0$ & $603(6.53)$ & $9(9.90)$ & & $9(15.79)$ & \\
\hline Normal weight 18.0-24.9 & $4611(50.0)$ & $45(49.45)$ & & $24(42.11)$ & \\
\hline Overweight 25-29.9 & $2508(26.17)$ & $25(27.47)$ & & $17(29.82)$ & \\
\hline Obese $30-39.9$ & $1392(15.08)$ & $12(13.19)$ & & $7(12.28)$ & \\
\hline Morbidly obese $>40$ & $206(2.23)$ & 0 & & 0 & \\
\hline Charlson score, mean (SD) & $3.6(2.9)$ & $4.0(3.2)$ & 0.16 & $3.9(2.9)$ & 0.41 \\
\hline INR category, n (\%) & & & 0.97 & & 0.58 \\
\hline $0.8-1.49$ & $6589(70.80)$ & $61(67.03)$ & & $39(68.40)$ & \\
\hline $1.5-2.99$ & $2005(21.5)$ & $20(22.00)$ & & $13(22.80)$ & \\
\hline$>3.1$ & $301(3.2)$ & $5(5.50)$ & & $1(1.80)$ & \\
\hline Partial thromboplastin time, mean (SD) & $41.3(21.8)$ & $46.3(27.8)$ & 0.03 & $47.0(32.5)$ & 0.06 \\
\hline Platelet category, n (\%) & & & 0.55 & & 0.21 \\
\hline$<20$ & $53(0.57)$ & 0 & & 0 & \\
\hline $20-49$ & $359(3.90)$ & $4(4.40)$ & & $2(3.51)$ & \\
\hline $50-100$ & $1002(10.9)$ & $13(14.29)$ & & $5(8.77)$ & \\
\hline$>100$ & $7670(82.10)$ & $74(84.61)$ & & $48(84.21)$ & $7670(82.10)$ \\
\hline Volume category, $\mathrm{n}(\%), \mathrm{mL}$ & & & $<0.0001$ & & $<0.0001$ \\
\hline 0 & $35(0.38)$ & $1(1.10)$ & & $1(1.75)$ & \\
\hline $1-1500$ & $8486(91.94)$ & $65(71.43)$ & & $38(66.67)$ & \\
\hline$>1500$ & $799(8.66)$ & $25(27.47)$ & & $18(31.58)$ & \\
\hline Bilateral procedure, $\mathrm{n}(\%)$ & $3796(41.12)$ & $19(20.89)$ & $<0.0001$ & $10(17.54)$ & $<0.0001$ \\
\hline Invasive or non-invasive mechanical ventilation, $\mathrm{n}(\%)$ & $1377(14.92)$ & $17(18.68)$ & 0.49 & $11(19.30)$ & 0.37 \\
\hline Needle passes category, n (\%) & & & 0.002 & & 0.001 \\
\hline 1 & $9190(99.57)$ & $89(97.80)$ & & 55 (96.49) & \\
\hline$\geq 2$ & $73(0.78)$ & $2(2.20)$ & & $2(3.51)$ & \\
\hline Supine, n (\%) & $473(5.12)$ & $6(6.59)$ & 0.51 & $6(10.53)$ & 0.08 \\
\hline
\end{tabular}

whom had any complication and four had pneumothorax; PTT values were missing in 467/9320 (5.0\%) patients, four of whom had any complication and four had pneumothorax; and platelet counts were missing in 236/9320 (2.5\%) patients, of whom none had any complications while two had pneumothorax.

The incidence of any complication was significantly higher for underweight patients compared with those of normal weight $(\mathrm{p}=0.02)$, in patients with higher PTT $(\mathrm{p}=0.04)$, those with $>1500 \mathrm{~mL}$ removed compared with $1-1500 \mathrm{~mL}(\mathrm{p}<0.0001)$, patients having unilateral compared with bilateral procedures $(\mathrm{p}<0.0001)$ and patients with more than one needle pass through the skin $(p=0.002$; table 1$)$. No other variables were associated with complications at the $\mathrm{p} \leq 0.1$ level.

Iatrogenic pneumothorax was significantly associated with $>1500 \mathrm{~mL}$ of fluid removed compared with $1-1500 \mathrm{~mL}$ $(\mathrm{p}<0.0001)$, unilateral procedures $(\mathrm{p}=0.001)$ and more than one needle pass through the skin $(p=0.001$; table 1$)$. The needle pass associations for both any complication and iatrogenic pneumothorax were completely driven by three needle passes as no patients had complications with two needle passes. Other variables associated with pneumothorax at the $\mathrm{p} \leq 0.1$ level are shown in table 1 . There were no associations between pneumothorax and Charlson score or positive pressure ventilation.

In the Poisson regression model, the effect of fluid volume $(\mathrm{mL})$ removed on the likelihood of REPE was significant $(p<0.0001,95 \%$ CI 1.0009 to 1.0026$)$. The association of volume removed with the likelihood of REPE is reflected by the fact that there were no events when no fluid was removed, four events $(0.05 \%)$ when $1-1500 \mathrm{~mL}$ was removed and six events $(0.75 \%)$ when $>1500 \mathrm{~mL}$ was removed. Details on the demographic characteristics for patients with REPE are shown in online supplementary E-table 1.

There were no significant associations between bleeding and demographic or clinical variables including INR, PTT and platelet counts. Details on the 17 cases of bleeding are shown in online supplementary E-table 2.

\section{Multivariable models of risk factors for any complications and pneumothorax}

In the multivariable models, every 1 unit increase in PTT was significantly associated with a $0.08 \%$ increased risk of any 
Table 2 Multiple regression analysis for any complication

\begin{tabular}{|c|c|c|c|c|}
\hline & \multirow[b]{2}{*}{ Incidence rate ratio } & \multicolumn{2}{|c|}{$95 \% \mathrm{Cl}$} & \multirow[b]{2}{*}{$\mathrm{p}$ Value } \\
\hline & & Lower & Upper & \\
\hline Continuous PTT* & 1.01 & 1.008 & 1.02 & 0.031 \\
\hline \multicolumn{5}{|l|}{ BMI category } \\
\hline Normal weight $18.0-24.9$ & Reference & & & \\
\hline Underweight $<18.0$ & 1.50 & 0.68 & 3.32 & 0.32 \\
\hline Overweight 25-29.9 & 0.95 & 0.56 & 1.60 & 0.85 \\
\hline Obese 30-39.9 & 0.89 & 0.46 & 1.72 & 0.73 \\
\hline Morbidly obese $>40$ & No events (constant) & & & \\
\hline \multicolumn{5}{|l|}{ Fluid volume removed category } \\
\hline 0 & No events (constant) & & & \\
\hline $1-1500 \mathrm{~mL}$ & Reference & & & \\
\hline$>1500 \mathrm{~mL}^{*}$ & 3.88 & 2.35 & 6.40 & $<0.0001$ \\
\hline Bilateral $^{*}$ & 0.45 & 0.27 & 0.77 & 0.003 \\
\hline \multicolumn{5}{|l|}{ Needle passes category } \\
\hline 1 & Reference & & & \\
\hline$\geq 2$ & 3.13 & 0.74 & 13.23 & 0.12 \\
\hline
\end{tabular}

complication $(p=0.03)$, while removing $>1500 \mathrm{~mL}$ of fluid was associated with 3.8 times higher risk $(\mathrm{p}<0.0001$; table 2$)$. Patients who underwent bilateral procedures experienced a $55 \%$ lower risk of any complication than those who underwent unilateral procedures $(\mathrm{p}=0.003)$.

In the pneumothorax model (table 3), underweight patients $(\mathrm{BMI}<18)$ were three times more likely to have had an iatrogenic pneumothorax $(\mathrm{p}=0.02)$ than normal weight patients. Bilateral procedures were associated with a $61 \%$ lower likelihood of pneumothorax $(p=0.01)$. Removing $>1500 \mathrm{~mL}$ fluid $(p<0.0001)$ and two or more needle passes $(p=0.03)$ were

Table 3 Multiple regression analysis for pneumothorax

\begin{tabular}{|c|c|c|c|c|}
\hline \multirow[b]{3}{*}{ Significant variable } & \multicolumn{4}{|c|}{ latrogenic pneumothorax } \\
\hline & \multirow[b]{2}{*}{ Incidence ratio } & \multicolumn{2}{|l|}{$95 \% \mathrm{Cl}$} & \multirow[b]{2}{*}{ p Value } \\
\hline & & Lower & Upper & \\
\hline Female & 0.93 & 0.51 & 1.70 & 0.82 \\
\hline \multicolumn{5}{|l|}{ BMI category } \\
\hline Normal weight 18.0-24.9 & Reference & & & \\
\hline Underweight $<18.0^{*}$ & 3.00 & 1.22 & 7.38 & 0.017 \\
\hline Overweight 25-29.9 & 1.17 & 0.59 & 2.33 & 0.65 \\
\hline Obese $30-39.9$ & 0.97 & 0.40 & 2.34 & 0.94 \\
\hline Morbidly obese $>40$ & No events (constant) & & & \\
\hline PTT & 1.01 & 1.00 & 1.02 & 0.059 \\
\hline \multicolumn{5}{|l|}{ Volume category $(\mathrm{mL})^{*}$} \\
\hline 0 & No events (constant) & & & \\
\hline $1-1500$ & Reference & & & \\
\hline$>1500$ & 4.87 & 2.52 & 9.42 & $<0.0001$ \\
\hline Supine & 1.37 & 0.51 & 3.67 & 0.53 \\
\hline Bilateral* & 0.39 & 0.19 & 0.81 & 0.011 \\
\hline \multicolumn{5}{|l|}{ Needle passes category } \\
\hline 1 & Reference & & & \\
\hline$\geq 2 * \dagger$ & 5.23 & 1.17 & 23.88 & 0.031 \\
\hline
\end{tabular}

each associated with approximately five times higher risk of pneumothorax (table 3).

\section{DISCUSSION}

The overall complication rate $(0.98 \%)$ and pneumothorax rate $(0.61 \%)$ for this large series of patients was lower than many published $^{\text {studies. }}{ }^{3} 8$ Available literature indicates that thoracentesis-associated pneumothorax occurs $0-19 \%$ of the time, while experienced performers achieve rates closer to 3.9\%. ${ }^{3}$ In 2010 the British Thoracic Society (BTS) published the only clinical guidelines for thoracentesis. ${ }^{8}$ Their consensus statement, not based on quality evidence, recommended against removing $>1500 \mathrm{~mL}$ of pleural fluid to reduce the risk of pneumothorax and REPE and avoiding procedures in patients on anticoagulants or with an INR of $\geq 1.5$. $^{8}$

In our study, significant factors associated with any complications were mostly driven by the frequency of iatrogenic pneumothorax. We cannot easily explain the relationship between increasing PTT and any complication, although the relationship was very small and we believe that it is not likely to be clinically meaningful because it was not specifically associated with bleeding complications. Further research is needed to investigate this relationship. Underweight patients were at higher risk for pneumothorax, probably because of the potential risk of underestimating the distance the needle entered through the skin and lack of relying on ultrasound measurements, shorter distances between the chest wall and the lung and/or less tolerance for invasive procedures due to poor nutritional status or overall health. It is not surprising that more needle passes would increase the risk of pneumothorax $(2 / 73,2.7 \%)$ because this is suggestive of a more difficult procedure. The association between larger fluid volumes removed and iatrogenic pneumothorax has also been seen in other studies. ${ }^{8}$ A likely explanation is that many of these patients may have had trapped lung before the procedure, and after large volumes were removed the areas of air/vacuum in the chest mimicked pneumothorax by appearing more prominent on the chest X-ray.

Bilateral procedures were associated with a lower incidence of pneumothorax. It seems reasonable that, if a unilateral procedure goes well (no obvious complications), the other pleural space can safely be drained. ${ }^{5}$ Despite lacking evidence, drainage of bilateral pleural effusions may be clinically warranted to optimise pulmonary function and wean patients off mechanical ventilation. The most logical explanation as to why bilateral procedures would have a lower risk of pneumothorax is that, if the first side procedure caused a complication, then attempts would be aborted on the other side. We do not know how many patients were planned for a bilateral procedure but only underwent a unilateral procedure. Additionally, it is possible that there are unknown patient factors that reduce the risk for the first side which are then conferred to the procedure on the other side. Contrary to other studies, our study showed that positive pressure ventilation was not associated with higher rates of pneumothorax. ${ }^{6} 7$ One meta-analysis reported a mean pneumothorax rate of $3.4 \%$ in 14 studies of patients undergoing thoracentesis on positive pressure ventilation, ${ }^{7}$ although our overall pneumothorax rate in patients receiving positive pressure ventilation was $0.8 \%$.

The cause of REPE continues to be a matter of debate. Reported incidence ranges from $0.2 \%$ to $14 \% 0^{12}$ and carries a disproportionately high mortality rate that approaches $20 \%{ }^{8}$ Our overall REPE rate was extremely low, occurring in only 10 cases $(0.01 \%)$ overall, with no deaths. ${ }^{14} 15$ We did discover a significantly higher risk of REPE when a higher volume was 
removed. Interestingly, previous publications have discussed the possible aetiology of REPE being related to pressure rather than to volume,$^{8}$ and some studies have failed to show a relationship between volume and REPE. ${ }^{12}{ }^{13}$ However, the overall numbers of REPE and patients in these studies were small and they may have been underpowered. Our operator routinely used the hand pumping method to remove fluid, generally avoided the use of vacuum bottles and did not use manometry. ${ }^{16}{ }^{17}$ Fluid removal was complete unless patients had worsening symptoms ${ }^{18}$ or the operator had the subjective sensation of increasingly negative intrapleural pressure, in which case the procedure was terminated early. Despite the relationship seen between volume removed and REPE in our study, volume is likely to play less of a role in the formation of REPE than a number of other factors which include pleural and lung elastance, chest cavity volume and degree of visceral pleural disease. Higher volumes may just put patients at a higher risk for REPE because of these other factors that accompany it. It is also possible that the major factor contributing to higher rates of REPE in large volume removal is the degree of negative pressure needed to aspirate all the fluid. Of note, many of our REPE complications were associated with non-inflammatory conditions that would not necessarily be expected to cause REPE such as liver disease, heart failure and nephrotic syndrome (table 2). Future REPE studies that control for diagnosis and fluid consistency (transudative or exudative) might prove illustrative, as would large randomised controlled trials to look at the safety of removing high volumes relative to intrathoracic pressures (with the use of real-time manometry).

The risk of bleeding in patients with coagulopathy has not been well established. In fact, the BTS guidelines are based on one retrospective series that showed the bleeding risk was lower in patients with coagulation studies below $2 \times$ the upper limit (INR < 1.5) and platelets $>50000 / \mu L^{8}{ }^{9}$ However, our data failed to prove a bleeding risk in patients with coagulopathy or low platelets.

Our study had several limitations. First, all thoracenteses were performed at a single site and were performed or supervised by one proceduralist. Further, only the proceduralist documented complications and may have been biased to underreport. We do not believe this to be the case, however, because all data were originally collected for quality assurance and performance improvement intent and without a plan to analyse or publish. In fact, the registry data included complications in a conservative manner, counting complications in areas where clinical certainty was not necessarily present. Additionally, all complications were tracked for $24 \mathrm{~h}$ after the procedure or until a clinical question was reconciled. Although it is possible that some complications may have been missed using this method, most thoracentesisrelated complications occur within $24 \mathrm{~h}$. While delayed complications were not systematically tracked to detect complications presenting to another institution, those patients presenting to CSMC with a delayed complication were detected as the procedure centre was routinely contacted for follow-up. Another limitation is related to limited statistical power with few REPE or bleeding complications such that haemothorax, site bleeding and haematomas needed to be combined into one category. This is important because we could not test for other confounding variables in REPE or bleeding models. Finally, many patients underwent more than one procedure across admissions. Although this was accounted for in the analysis by controlling for clustering at the patient level, the overall rate of complications per patient (not per procedure) was $1.86 \%$ including a $1.23 \%$ pneumothorax rate. These per-person rates may be more comparable to studies conducted among patients who only received unilateral single procedures.

\section{CONCLUSIONS}

This series of thoracenteses had a very low overall complication rate, serving as yet another example that high procedure volume and operator experience are major determinants in outcomes. ${ }^{19-22}$ The results also suggest that many current clinical guidelines and practice patterns may not reflect evidence-based best practices, while others do appear to have statistical merit. Ultimately, it is our strong recommendation that decisions regarding whether or not to perform bilateral procedures on patients receiving positive pressure ventilation, how much fluid to remove and the need for blood product transfusion should be left in the hands of fully informed and experienced clinicians. It may not be possible to achieve our same results in centres with a lower number of procedures and expertise.

Acknowledgements We acknowledge Drs Douglas Vaughan and Kevin O'Leary for their support and encouragement of this work. We would also like to thank Julian Martinez and the Cedars-Sinai Enterprise Information Systems Department for assistance with our data query.

Contributors MJA contributed to the study conception and design, performance of all the thoracenteses, data acquisition and writing, editing and final approval of the manuscript. BTR contributed to the study conception and design, data acquisition and writing, editing and final approval of the manuscript. IS contributed to the data acquisition, statistical analysis, editing and final approval of the manuscript. JF contributed to the study design, data acquisition, statistical analysis, editing and final approval of the manuscript. JHB contributed to the study conception and design, data acquisition, statistical analysis and writing, editing and final approval of the manuscript.

Funding The contributions of JS, JF and JHB to this project were partly supported by grant R18HS021202-01 from the Agency for Healthcare Research and Quality (AHRQ). AHRQ had no role in the preparation, review or approval of the manuscript.

\section{Competing interests None.}

Ethics approval Ethics approval was obtained from Cedars-Sinai and Northwestern University Institutional Review Board.

Provenance and peer review Not commissioned; externally peer reviewed.

\section{REFERENCES}

1 Light RW. Pleural diseases. 5th edn. Philadephia: Lippincott Williams \& Wilkins, 2007.

2 Owings MF, Kozak LJ. Ambulatory and inpatient procedures in the United States, 1996. Vital Health Stat 13 1998;(139):1-119.

3 Gordon CE, Feller-Kopman D, Balk EM, et al. Pneumothorax following thoracentesis: a systematic review and meta-analysis. Arch Intern Med 2010;170:332-9.

4 Mercaldi CJ, Lanes SF. Ultrasound guidance decreases complications and improves the cost of care among patients undergoing thoracentesis and paracentesis. Chest 2013;143:532-8.

5 Puchalski JT, Argento AC, Murphy TE, et al. Etiologies of bilateral pleural effusions. Respir Med 2013;107:284-91.

6 Azoulay E. Pleural effusions in the intensive care unit. Curr Opin Pulm Med 2003:9:291-7.

7 Goligher EC, Leis JA, Fowler RA, et al. Utility and safety of draining pleural effusions in mechanically ventilated patients: a systematic review and meta-analysis. Crit Care 2011;15:R46.

8 Havelock T, Teoh R, Laws D, et al. Pleural procedures and thoracic ultrasound: British Thoracic Society pleural disease guideline 2010. Thorax 2010;65(Suppl 2): ii61-76.

9 McVay PA, Toy PT. Lack of increased bleeding after paracentesis and thoracentesis in patients with mild coagulation abnormalities. Transfusion 1991;31:164-71.

10 Deyo RA, Cherkin DC, Ciol MA. Adapting a clinical comorbidity index for use with ICD-9-CM administrative databases. J Clin Epidemiol 1992;45:613-19.

11 Romano PS, Roos LL, Jollis JG. Adapting a clinical comorbidity index for use with ICD-9-CM administrative data: differing perspectives. J Clin Epidemiol 1993:46:1075-9.

12 Echevarria C, Twomey D, Dunning J, et al. Does re-expansion pulmonary oedema exist? Interact Cardiovasc Thorac Surg 2008;7:485-9.

13 Feller-Kopman D, Berkowitz D, Boiselle $P$, et al. Large-volume thoracentesis and the risk of reexpansion pulmonary edema. Ann Thorac Surg 2007;84:1656-61. 
14 Iqbal M, Multz AS, Rossoff LJ, et al. Reexpansion pulmonary edema after VATS successfully treated with continuous positive airway pressure. Ann Thorac Surg 2000;70:669-71.

15 Tariq SM, Sadaf T. Images in clinical medicine. Reexpansion pulmonary edema after treatment of pneumothorax. N Engl J Med 2006;354:2046.

16 Feller-Kopman D. Therapeutic thoracentesis: the role of ultrasound and pleural manometry. Curr Opin Pulm Med 2007;13:312-18.

17 Feller-Kopman D. Point: should pleural manometry be performed routinely during thoracentesis? Yes. Chest 2012;141:844-5.

18 Feller-Kopman D, Walkey A, Berkowitz $D$, et al. The relationship of pleural pressure to symptom development during therapeutic thoracentesis. Chest 2006;129:1556-60.
19 Dudley $\mathrm{RA}$, Johansen $\mathrm{KL}$, Brand $\mathrm{R}$, et al. Selective referral to high-volume hospitals: estimating potentially avoidable deaths. JAMA 2000;283:1159-66.

20 Hannan EL, Kilburn H Jr, Bernard H, et al. Coronary artery bypass surgery: the relationship between inhospital mortality rate and surgical volume after controlling for clinical risk factors. Med Care 1991;29:1094-107.

21 Jollis JG, Peterson ED, DeLong ER, et al. The relation between the volume of coronary angioplasty procedures at hospitals treating Medicare beneficiaries and short-term mortality. N Engl J Med 1994;331:1625-9.

22 Showstack JA, Rosenfeld KE, Garnick DW, et al. Association of volume with outcome of coronary artery bypass graft surgery. Scheduled vs nonscheduled operations. JAMA 1987;257:785-9. 